\title{
Association of Dengue Virus and Leptospira Co-Infections with Malaria Severity
}

\author{
Rajendra Mandage, ${ }^{1}$ Charandeep Kaur, ${ }^{1}$ Atreyi Pramanik, ${ }^{1}$ Vinod Kumar, ${ }^{2}$ Parul Kodan, Adarsh Singh, \\ Sounak Saha, Shivam Pandey, Naveet Wig, Ravindra Mohan Pandey, Manish Soneja, Pragyan Acharya
}

\begin{abstract}
Plasmodium infections are co-endemic with infections caused by other agents of acute febrile illnesses, such as dengue virus (DENV), chikungunya virus, Leptospira spp., and Orientia tsutsugamushi. However, coinfections may influence disease severity, treatment outcomes, and development of drug resistance. When we analyzed cases of acute febrile illness at the All India Institute of Medical Sciences, New Delhi, India, from July 2017 through September 2018, we found that most patients with malaria harbored co-infections (Plasmodium mixed species and other pathogens). DENV was the most common malaria co-infection ( $44 \%$ of total infections). DENV serotype 4 was associated with mild malaria, and Leptospira was associated with severe malaria. We also found the presence of $P$. knowlesi in our study population. Therefore, in areas with a large number of severe malaria cases, diagnostic screening for all 4 DENV serotypes, Leptospira, and all Plasmodium species should be performed.
\end{abstract}

Tn tropical countries, including India, acute febrile Iillnesses (AFIs) constitute a group of infections with similar manifestations, such as fever, malaise, body aches, chills, hepatic and renal dysfunction, and central nervous system effects. The causative agents of AFI can be bacterial (e.g., Orientia tsutsugamushi, Leptospira, and Salmonella enterica serovar Typhi), parasitic (protozoans of the apicomplexa family), or viral (e.g., dengue virus [DENV], chikungunya virus [CHIKV], influenza A[H1N1] virus) (1-4). Distinguishing between the causative agents of AFIs can be difficult. In tropical climates, several AFI pathogens,

Author affiliations: All India Institute of Medical Sciences, New Delhi, India (R. Mandage, C. Kaur, A. Pramanik, P. Kodan, S. Pandey, N. Wig, R.M. Pandey, M. Soneja, P. Acharya); Nehru Shatabdi Chikitsalaya, Singrauli, India (V. Kumar); Indian Institute of Technology, Kharagpur, West Bengal, India (A. Singh, S. Saha)

DOI: https://doi.org/10.3201/eid2608.191214 such as malaria parasites, DENV, and CHIKV, occur in the same areas and during the same seasons (5), making it possible that $>1$ pathogen can infect the same person. Indeed, recent retrospective analyses based on persons hospitalized with an AFI have uncovered malaria co-infections with dengue, chikungunya, and leptospirosis in different populations across the world (6-14).

Despite the increasing realization that co-infections may contribute to the course and outcome of malaria, only a few studies have investigated the prevalence and nature of co-infections (14-20), which limits our ability to manage and understand AFIs, as follows. First, we do not know the spectrum of infections that a person with an AFI may harbor, leading to inadequate drug therapy. Treatment strategies based on diagnosis of a single pathogen may lead to inadvertent exposure of the undetected pathogen to antimicrobial agents, thereby contributing to generation of antimicrobial-resistant species. Second, lack of adequate data on co-infections in clinical and field settings can misdirect the field of drug and vaccine development. Pathogens such as malaria parasites, DENV, and Orientia spp. have host immune-modulatory effects (21). Therefore, co-infections can aid or antagonize each other in terms of evading host immune responses. These interactions may have major effects on immune responses to vaccine candidates and need to be known during design of effective vaccination strategies (22). Third, we do not know how interactions of co-infecting pathogens lead to diverse disease outcomes affecting organ function and ultimately mortality. In India, the prevalence of malaria parasites, DENV, and CHIKV resembles the global prevalence and co-endemicity of these pathogens (5).

\footnotetext{
${ }^{1}$ These authors contributed equally to this article.
}

${ }^{2}$ Work was conducted at the All India Institute of Medical Sciences, New Delhi. 
Malaria infections in India are reportedly caused by Plasmodium falciparum, P. vivax, P. ovale, and P. malariae (23). Several studies have also reported the occurrence of $P$. vivax severe malaria in India as well as in Southeast Asia and South America (23-25). Our objective with this study was to define the spectrum of co-infections in patients with an AFI associated with malaria admitted to the All India Institute of Medical Sciences, New Delhi, India, a tertiary care research hospital.

\section{Materials and Methods}

\section{Study Participants and Sample Collection}

For our prospective study, we recruited patients with an AFI (history of fever, i.e., temperature $>38^{\circ} \mathrm{C}$ that had persisted for $\geq 2$ days without an identified source) from the Department of Medicine at All India Institute of Medical Sciences from July 2017 through
September 2018. Every admitted consenting AFI patient was tested by PCR for all 5 Plasmodium species $(P$. falciparum, P. vivax, P. malaria, P. ovale, and P. knowlesi), DENV, CHIKV, O. tsutsugamushi, and Leptospira. The study was approved by the institute research ethics committee (reference no. IEC-55/07.10.2016, RP7/2017).

For each participant, we collected information about geographic location (Figure) and completed a standard questionnaire (including demographic information, history, general physical examination findings, systemic examination findings, and clinical investigation findings). To determine presumptive clinical diagnoses and treatments, we reviewed medical chart records corresponding to each participant.

All patient data were anonymized to protect confidentiality. Blood samples were collected and subjected to microscopy, rapid diagnostic testing, and PCR analysis for all 5 pathogens $(5$ species of

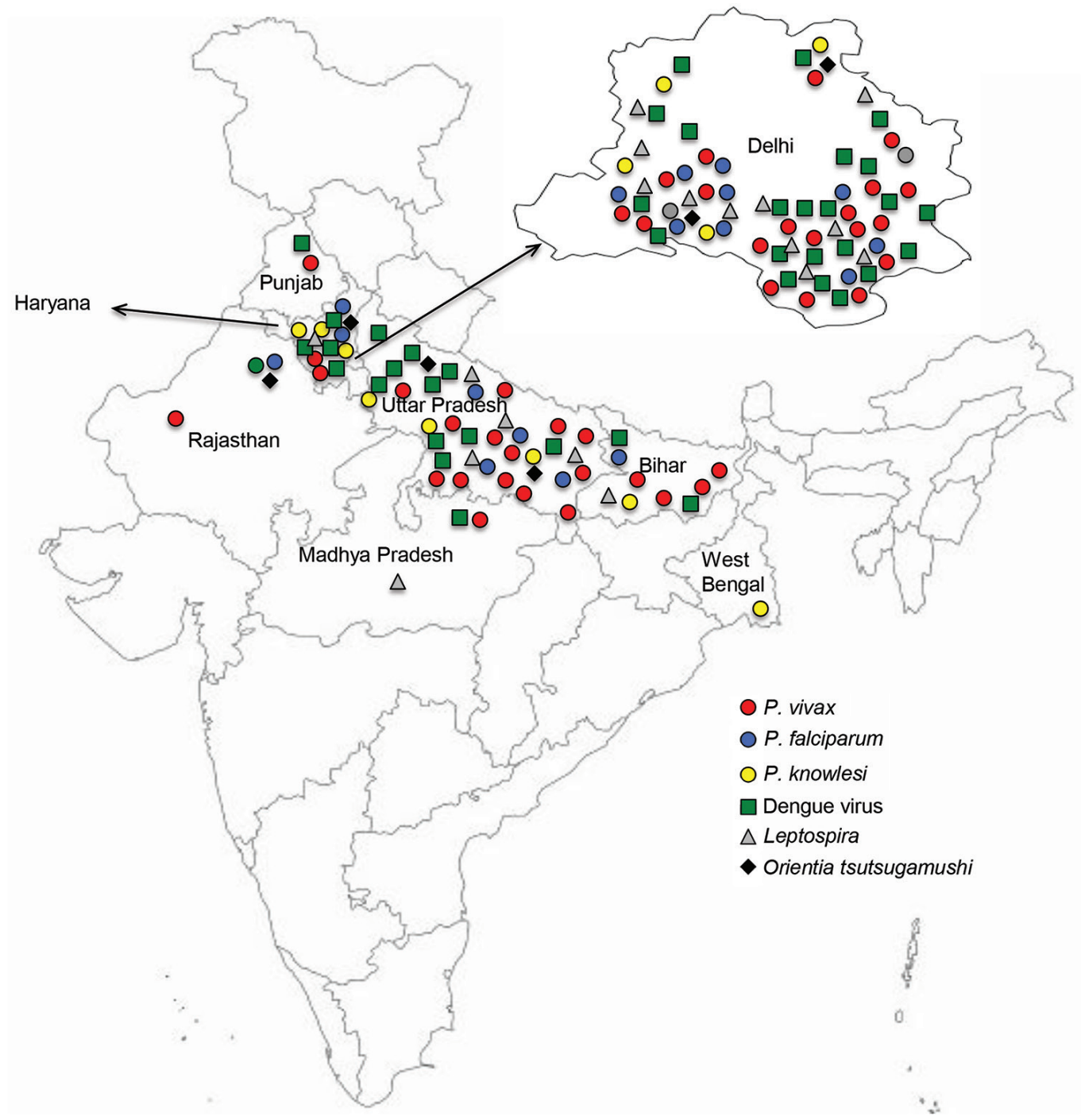

Figure. Locations of malaria patients with co-infections, India, July 2017-September 2018. Close-up view of Delhi state is provided. 
Plasmodium, DENV, CHIKV, Leptospira, and Orientia). Typhoid testing was not conducted for patients with no abdominal pain or diarrhea. None of the patients recruited for this study showed indications for typhoid testing.

For microscopic examinations, we used peripheral blood smears (Giemsa-stained thick and thin smears) and a 3-band rapid diagnostic test kit (SD Bioline Malaria Ag Pf/Pan kit; Standard Diagnostics, Inc., https://www.alere.com/en/home.html). The rapid diagnostic test detects antigens specific to histidine-rich protein II from $P$. falciparum and panPlasmodium lactate dehydrogenase from $P$. vivax, $P$. malariae, or P. ovale.

Patients positive for malaria by PCR were classified as having severe malaria according to World Health Organization 2015 guidelines (https:// www.who.int/docs/default-source/documents/ publications/gmp/guidelines-for-the-treatment-ofmalaria-eng.pdf?sfvrsn=a0138b77_2). These guidelines define severe malaria as creatinine level $>3$ $\mathrm{mg} / \mathrm{dL}$, bilirubin level $>3 \mathrm{mg} / \mathrm{dL}$, bicarbonate level $<15 \mathrm{mmol} / \mathrm{L}$, hemoglobin level $<7 \mathrm{~g} / \mathrm{dL}$ for adults and $<5 \mathrm{~g} / \mathrm{dL}$ for children, parasite count $10 \%$, hypoglycemia <2.2 mM, substantial bleeding, impaired consciousness, shock, prostration (defined as myalgia and arthralgia), multiple convulsions, and pulmonary edema) (26). The remaining patients were classified as having mild malaria.

\section{DNA Extraction and PCR Analyses}

From participating AFI patients, we collected $5 \mathrm{~mL}$ of venous blood into EDTA tubes for PCR analysis. We extracted DNA from whole blood by using a QiaAmp DNA Mini Kit (QIAGEN, https://www.qiagen.com) according to the manufacturer's instructions. To detect DENV and CHIKV, we extracted RNA from TRIzol by using the isopropanol method, and we synthesized complementary DNA from RNA by using a Verso cDNA Synthesis Kit (Thermo Fisher Scientific, https:/ / www.thermofisher.com) according to the manufacturer's recommendations. We analyzed all samples for the presence of all 5 human Plasmodium species, O. tsutsugamushi, Leptospira, DENV, and CHIKV. All samples were also subjected to microscopy and rapid diagnostic testing (for PfHRP2 and PvLDH genes) for malaria diagnosis. The diagnosis of DENV and its serotypes was conducted by using serotype-specific PCR primers. The presence of other infectious agents, such as O. tsutsugamushi, Leptospira, and CHIKV was detected by PCR (Appendix Table 1, https:/ / wwwnc.cdc.gov/ EID/article/26/8/19-1214-App1.pdf). Randomly selected representative PCR products were subjected to Sanger sequencing to confirm species identity (Appendix Table 2).

We categorized the types of infections or combination of infections in a person as monoinfections, mixed infections, or co-infections. Monoinfections are defined as infections with 1 species of Plasmodium,

\begin{tabular}{|c|c|}
\hline Pathogen & No. co-infections \\
\hline \multicolumn{2}{|l|}{ All Plasmodium-positive infections, $\mathrm{n}=66$} \\
\hline P. falciparum alone & 10 \\
\hline$P$. vivax alone & 34 \\
\hline P. knowlesi alone & 5 \\
\hline$P$. vivax $+P$. knowlesi & 4 \\
\hline P. falciparum + P. vivax & 10 \\
\hline$P$. falciparum $+P$. knowlesi & 1 \\
\hline$P$. falciparum $+P$. vivax $+P$. knowlesi & 2 \\
\hline \multicolumn{2}{|l|}{ Plasmodium + bacteria co-infections, $\mathrm{n}=17$} \\
\hline Plasmodium + O. tsutsugamushi & 5 \\
\hline Plasmodium + Leptospira & 11 \\
\hline Plasmodium + Leptospira + O. tsutsugamushi & 1 \\
\hline \multicolumn{2}{|l|}{ Plasmodium + DENV co-infections, $\mathrm{n}=40$} \\
\hline Plasmodium + DENV, all serotypes & 40 \\
\hline Plasmodium + DENV-1 & 8 \\
\hline Plasmodium + DENV-3 & 5 \\
\hline Plasmodium + DENV-4 & 20 \\
\hline Plasmodium + DENV-1 + DENV-3 & 1 \\
\hline Plasmodium + DENV-1 + DENV-4 & 2 \\
\hline Plasmodium + DENV-3 + DENV-4 & 1 \\
\hline Plasmodium + DENV-1 + DENV-4 + DENV-3 & 3 \\
\hline \multicolumn{2}{|l|}{ Plasmodium + DENV + bacteria co-infections, $\mathrm{n}=11$} \\
\hline Plasmodium + DENV + Leptospira & 8 \\
\hline Plasmodium + DENV+ O. tsutsugamushi & 2 \\
\hline Plasmodium + DENV + Leptospira + O. tsutsugamushi & 1 \\
\hline
\end{tabular}


mixed infections with $>1$ Plasmodium species, and co-infections with Plasmodium species and other bacterial or viral infections.

\section{Determination of Patient Locations and Construction of Map of India}

We were able to retrieve location data for 82 patients. We constructed a map of India based on the official maps provided by the Survey of India (http://www. surveyofindia.gov.in/pages/display/235-politicalmap-of-india), as described previously (26). In brief, we downloaded an India map shapefile (http:/ / www. indianremotesensing.com/2017/01/Download-Indiashapefile-with-kashmir.html) and generated the final image by using Microsoft PowerPoint (https://www. microsoft.com) to map each patient to their local area. In addition, the 12 patients with $P$. knowlesi infection were asked to answer questions about time of malaria infection (as recorded in our dataset), travel outside India in 2 years preceding the malaria infection, visits from abroad by friends/relatives, and any previous malaria infections (possibility of recurrence/relapse).

\section{Statistical Analyses}

We recorded data on a predesigned form and managed the data in a Microsoft Excel spreadsheet and checked all entries for possible manual errors. We summarized categorical variables by frequency (\%) and age as means. We used $\chi^{2}$ or Fisher exact tests, or both, as appropriate, to compare frequencies between 2 groups and the Student $t$-test to compare age distribution between 2 groups. We evaluated accuracy of microscopy and rapid diagnostic testing methods by using PCR as a reference for malaria diagnosis. For each of the 2 tests, we computed sensitivity, specificity, positive predictive value, negative predictive value, positive likelihood ratio, and negative likelihood ratio by using PCR as a reference. We also computed 95\% CIs for each measure computed to determine the strength of association of various co-infections with malaria severity. We used bivariate and multivariate logistic regression methods to determine the odds ratio $(95 \% \mathrm{CI})$ for each co-infection by using Stata version 15.0 statistical software (https://www.stata.com).
We considered $p<0.05$ to be statistically significant. We created a patient baseline characteristics table by using the $\mathrm{R}$ version 3.4.3 package tableone (27). The tableone package summarizes categorical data in the form of counts and percentages and summarizes continuous data in the form of means and SDs.

\section{Results}

\section{Spectrum of Co-infections and Plasmodium Mixed Species Infections in Patients with Malaria}

We analyzed the prevalence of various co-infections and Plasmodium mixed-species infections in the 66 Plasmodium-positive samples (Table 1 ). P. vivax accounted for most $(76 \%)(50 / 66)$ infections, whereas P. falciparum accounted for $35 \%$ (23/66). P. knowlesi was detected in $18 \%$ (12/66) of infections (Table 1); $P$. malariae and $P$. ovale were not detected in our study.

From the 66 Plasmodium-positive patients, 40 (60\%) samples indicated a DENV co-infection with or without other co-infecting pathogens, and 29 (44\%) indicated exclusive Plasmodium/DENV co-infections. Plasmodium co-infections with bacteria were found for $16(25 \%)$ patients: Leptospira infections for $11(17 \%)$ of the 66 and O. tsutsugamushi for 5 (8\%) (Table 1).

Mapping indicated that locations of the malaria patients in our study spanned the entire northern region of India, including the states of Rajasthan, Haryana, Punjab, Delhi, Uttar Pradesh, Bihar, and West Bengal (Figure). Patients with P. knowlesi infection originated from Delhi, Haryana, Uttar Pradesh, and West Bengal. Most patients with dengue infections originated from Delhi and Uttar Pradesh. Of the 12 patients with $P$. knowlesi infection, 5 had not traveled abroad or had direct contact with any visitors from abroad for at least 2 years before admission. No information was available for the remaining 7 patients (Appendix Table 3).

\section{Patient Baseline Characteristics}

Detailed hematologic and biochemical parameters for all patients were retrieved from medical records (Table 2; Appendix Table 4). Differences between severe and mild malaria patients were found in hemoglobin

\begin{tabular}{|c|c|c|}
\hline Parameter & Mild disease, mean ( \pm SD), $\mathrm{n}=33 \dagger$ & Severe, mean $( \pm \mathrm{SD}), \mathrm{n}=33 \ddagger$ \\
\hline Hemoglobin, g/dL & $12.11( \pm 3.22)$ & $9.89( \pm 2.96)$ \\
\hline Hematocrit, \% & $36.45( \pm 9.48)$ & $29.93( \pm 8.95)$ \\
\hline Platelets, $\times 10^{3} / \mu \mathrm{L}$ & $87.00( \pm 54.73)$ & $76.69( \pm 66.24)$ \\
\hline Leukocytes, $\times 10^{3}$ cells $/ \mu \mathrm{L}$ & $6.07( \pm 3.20)$ & $10.53( \pm 6.98)$ \\
\hline Erythrocytes, $\times 10^{6}$ cells $/ \mu \mathrm{L}$ & $4.26( \pm 1.34)$ & $3.69( \pm 0.95)$ \\
\hline Creatinine, $\mathrm{mg} / \mathrm{dL}$ & $0.90( \pm 0.41)$ & $3.37( \pm 3.41)$ \\
\hline
\end{tabular}


levels (9.89 g/dL vs. $12.11 \mathrm{~g} / \mathrm{dL})$, hematocrit $(29.93 \%$ vs. $36.45 \%)$, platelet counts $\left(76.69\right.$ vs. $\left.87 \times 10^{3} / \mu \mathrm{L}\right)$, leukocyte counts (10.53 vs. 6.07) $\times 10^{3}$ cells $\left./ \mu \mathrm{L}\right)$, and creatinine levels (3.37 vs. 0.90). Each group contained 26 male patients; mean age for severe malaria patients was 28 years and for mild malaria patients was 32 years.

\section{Association of Co-infecting Pathogens with Malaria Severity}

We found that co-infection with DENV serotype 4 (DENV-4) was associated with mild malaria (adjusted odds ratio [aOR] 0.3, 95\% CI 0.4-5.0), whereas infection with Leptospira (aOR 1.6, 95\% CI 0.4-6.8) or O. tsutsugamushi (aOR 1.1, 95\% CI 0.1-7.8) was associated with severe malaria. P. vivax or P. knowlesi monoinfection was also associated with severe malaria (aOR 2.5, 95\% CI 0.9-7.2) (Table 3). Other categories of Plasmodium mixed-species infections did not show any strong association with malaria severity (Appendix Table 5). However, the species of Plasmodium may confound some of these analyses.

\section{Relative Performance of Malaria Diagnostic Procedures} All 99 patients were tested for Plasmodium species by microscopy (8 positive results), rapid diagnostic testing (26 positive), and PCR (66 positive) (Table 4). Almost $50 \%$ of the P. vivax infections escaped detection by both microscopy and rapid testing. $P$. knowlesi was detectable solely by PCR. In addition, rapid diagnostic testing was able to detect only 1 of 18 Plasmodium mixed-species infections (Table 4). The diagnostic performance of microscopy and rapid diagnostic testing was calculated, and each was found to have poor sensitivity compared with PCR (Appendix Table 6).

\section{Discussion}

Among patients hospitalized with AFI at the All India Institute of Medical Sciences during July 2017-
September 2018, the major circulating Plasmodium species was $P$. vivax and malaria/DENV co-infections predominated. A high number of severe malaria cases reported to the institute are from northern India. Among the 5 Plasmodium species known to infect humans, in our study population we detected P. falciparum, P. vivax, and P. knowlesi but found no evidence of P. malariae or P. ovale. Most AFI patients in this study originated from northern India across the states of Rajasthan, Haryana, Punjab, Delhi, Uttar Pradesh, Bihar, and West Bengal. The burden of co-infecting pathogens in patients with malaria was revealed by a combination of complete blood work (peripheral blood smear analysis, rapid diagnostic testing, serum renal and liver function testing) and in-depth molecular assays (PCR amplification of Plasmodium speciesspecific genes followed by Sanger sequencing). We found a very high percentage of Plasmodium/DENV co-infections in our study population. This finding can be partly attributed to the highly sensitive PCR diagnostic methods used.

A recent meta-analysis of the prevalence of DENV/ Plasmodium/CHIKV co-infections spanning 7 geographic regions (southern Asia, Africa, Southeast Asia, South America, North America, the Caribbean, and the Middle East) showed that DENV/ Plasmodium co-infections have been reported in 19 countries, including India; DENV/CHIKV co-infections have been reported in 24 countries including India; CHIKV/Plasmodium co-infections have been reported in 6 countries with only a single coinfection reported from India; and DENV/CHIKV/ Plasmodium co-infections have been reported in 3 countries (5). According to that meta-analysis, the average reported prevalence of DENV/Plasmodium co-infection in India is $\approx 6.5 \%$, which is much lower than that detected by our study. However, a more detailed analysis from the eastern India state of Odisha

\begin{tabular}{|c|c|c|c|c|c|}
\hline \multirow[b]{2}{*}{ Co-infections } & \multicolumn{3}{|c|}{ No. malaria cases } & \multirow{2}{*}{$\begin{array}{l}\text { Unadjusted OR } \\
(95 \% \mathrm{Cl})\end{array}$} & \multirow{2}{*}{$\begin{array}{c}\text { Adjusted OR } \\
(95 \% \mathrm{Cl})\end{array}$} \\
\hline & Severe, $n=33$ & Mild, $\mathrm{n}=33$ & $p$ value & & \\
\hline DENV & & & $<0.08$ & & \\
\hline Neg for DENV & 14 & 12 & & Referent & Referent \\
\hline Pos for DENV-4 & 6 & 14 & & $0.34(0.1-1.2)$ & $0.3(0.4-5.0)$ \\
\hline Pos for other DENV serotypes: $1,3,1+3,4+3$ & 13 & 7 & & $1.6(0.5-2.5)$ & $1.4(0.4-4.9)$ \\
\hline Leptospira & & & $\leq 0.5$ & & \\
\hline Absent & 26 & 29 & & & \\
\hline Present & 7 & 4 & & $1.9(0.5-7.4)$ & $1.6(0.4-6.8)$ \\
\hline Orientia tsutsugamushi & & & $\leq 0.5$ & & \\
\hline Absent & 30 & 31 & & Referent & Referent \\
\hline Present & 3 & 2 & & $1.6(0.2-9.9)$ & $1.1(0.1-7.8)$ \\
\hline Malaria parasite types & & & $\leq 0.1$ & & \\
\hline Plasmodium mixed infections & 17 & 10 & & Referent & Referent \\
\hline P. vivax/P. knowlesi monoinfection & 16 & 23 & & $2.4(0.9-6.7)$ & $2.5(0.9-7.2)$ \\
\hline
\end{tabular}

*Bivariate and multivariate logistic regression analysis was used to determine the strength of association of various co-infections and mixed malaria infections with malaria severity. DENV, dengue virus.; neg, negative; pos, positive; OR, odds ratio. 
Table 4. Summary of detection of Plasmodium species by RDT, microscopy and PCR, India, July 2017-September 2018*

\begin{tabular}{lccc}
\hline Parasites & RDT, no. (\%) & Microscopy, no. (\%) & PCR, no. (\%) \\
\hline Plasmodium negative & $73(71.7)$ & $90(90.9)$ & $33(33.3)$ \\
\hline Plasmodium positive & $26(28.3)$ & $9(9.09)$ & $66(66.7)$ \\
P. falciparum & $6(6.06)$ & $2(2.02)$ & $10(10.1$ \\
P. vivax & $14(14.1)$ & $7(7.07)$ & $34(34.3)$ \\
P. ovale & 0 & 0 & 0 \\
P. malariae & 0 & 0 & 0 \\
P. knowlesi & 0 & 0 & $5(5.05)$ \\
\hline Mixed Plasmodium & $1(1.01)$ & 0 & $17(17.2)$ \\
Pan-Plasmodium & $7(7.07)$ & 0 & 0 \\
\hline *Percentages are calculated out of all AFI samples $(\mathrm{n}=99)$. RDT, rapid diagnostic test. & & \\
\hline
\end{tabular}

shows that this percentage can vary within a year, depending on season, and the highest reported prevalence of DENV/Plasmodium co-infections from this region was $31.8 \%$ during September-October, an observation similar to ours (28).

Although awareness of Plasmodium/DENV coinfections is increasing, little information is available about Plasmodium/Leptospira or Plasmodium/O. tsutsugamushi co-infections $(13,29)$. This lack of information is concerning because our study suggests that Plasmodium/Leptospira co-infections are associated with severe malaria. Prevalence data for co-infections with these pathogens are limited. We emphasize the need for such information because although these pathogens are carried by different vectors, they coexist in the same geoclimactic habitats that combine a warm, moist environment with dense vegetation and poor socio-economic development $(13,29)$. The presence of one co-infecting pathogen can influence disease outcomes, treatment outcomes, development of immunity, or drug resistance with regard to infections caused by the other co-infecting pathogen. One example is the predisposition for bacteremia to develop in persons with malaria (30).

In most malaria-endemic settings, malaria is still diagnosed by microscopic examination of Giemsastained peripheral blood smears and rapid diagnostic testing for parasite antigen. The rapid test is specifically designed to detect $P$. falciparum and $P$. vivax and is extensively used because of its speed and simplicity. For microscopy, diagnostic success depends on the skill of the technicians who observe the peripheral blood smears. We found that rapid tests and microscopy missed most of the $P$. vivax-positive malaria cases and all P. knowlesi cases and detected only 1 of 18 Plasmodium mixed-species infections. This finding clearly shows the limitations of rapid testing and microscopy for comprehensive detection of malaria parasites, which have been independently observed in several studies and attributed to deletions in the HRP2 and HRP3 genes in the specific case of $P$. falciparum infection (31-34). This problem is well recognized for healthcare workers and researchers working toward malaria elimination all over the world. Although the rapid diagnostic test for malaria has been shown to be better than microscopic examination of Giemsa-stained peripheral blood smears, PCR has been shown to be far superior to rapid testing for diagnosing low-parasitemia malaria infections (35). Our observations were similar; PCR was most sensitive, followed by rapid testing and then microscopy. However, rapid tests have low success rates in areas of low transmission intensities and give rise to a high proportion of false negatives (36). In addition, rapid tests fail to detect infections with emerging pathogens, such as the simian parasite species P. knowlesi and P. cynomolgi, both known to infect humans (37). Although recent reports highlight the improvement of rapid tests for $P$. knowlesi detection by use of a cross-reacting pan-parasite lactate dehydrogenase feature, we were unable to detect $P$. knowlesi by using a pan-parasite lactate dehydrogenase-containing rapid test, possibly because of low parasitemia, below the detection limit of the rapid test (38). P. knowlesi, which was previously believed to be localized to Southeast Asia, has now been reported from various parts of the world as single case reports of travelers' infections from areas including Oceania, Europe, and the Middle East (39-41). From India, P. knowlesi infection has been reported from the Andaman and Nicobar Islands in the context of drug resistance and in a recent study by our group in the context of acute kidney injury $(26,42)$. Historically, P. knowlesi infection was discovered as a naturally occurring human infection in Malaysia in 1965 (43). The accurate diagnosis of $P$. knowlesi by use of PCR took $\approx 40$ years from its initial discovery and gave a preliminary indication of the burden of this zoonosis in Sarawak, Malaysia (44). Until this point, P. knowlesi as a human infection was frequently misdiagnosed as $P$. vivax, $P$. malariae, or $P$. falciparum infection.

To assess whether the infections originated locally, we surveyed the $P$. knowlesi patients in our study group for the possibility of travel abroad or 
interaction with visitors from abroad within their family. The patients who responded to our questionnaire do not appear to have traveled abroad or to have had direct contact with anyone visiting them from abroad, suggesting local presence of $P$. knowlesi. However, unknown sources of travel from Southeast Asia, a neighbor to India, cannot be ruled out. Currently, testing for P. knowlesi is not included in diagnostic procedures in India, irrespective of diagnostic method (microscopy, rapid diagnostic test, or PCR), because it has not been widely reported. However, India is known to harbor both the potential vector for P. knowlesi, the Anopheles dirus mosquito, as well as the reservoir, pig-tailed macaques (Macaca nemestrina), thereby making India a potential ecosystem for the proliferation of this zoonotic Plasmodium species (45). Furthermore, the populations of Macaca mulatta macaques and related species have recently expanded in northern India, particularly in the state of Uttar Pradesh, which may explain the appearance of $P$. knowlesi in our study population representative of these areas, whereas it was not reported earlier (45). A recent report has also demonstrated the presence of $P$. falciparum parasites in monkey populations from India, indicating freely occurring undetected zoonotic transfer of the malaria parasites across reservoirs and hosts. Therefore, healthcare workers and national programs should incorporate all species of malaria parasites known to infect humans, in their diagnostic portfolio.

In conclusion, our study clearly showed that microscopy and rapid diagnostic testing gave falsenegative results for most mixed-species infections and completely missed P. knowlesi infections, coinfections and mixed Plasmodium infections were highly prevalent in patients with malaria, Plasmodium/DENV co-infections were the most common co-occurring pathogens in our study population, $P$. knowlesi infections were present in India, Plasmodium/DENV4 co-infections were associated with mild malaria, and Plasmodium/Leptospira infections were associated with severe malaria. Although the ORs support the above findings, the $95 \%$ CIs for these associations were wide. CIs reflect the uncertainty of the estimated effect, and wider intervals suggest greater uncertainty. The wide 95\% CIs in this study suggest that although trends were observed, additional data points are needed to determine the effect size of these associations. Wider prevalence studies investigating malaria co-infections are needed.

The government of India has recently declared a goal of malaria elimination by 2030 , which will be a major step toward global malaria eradication because India serves as a major Plasmodium reservoir, contributing to almost $4 \%$ of malaria-related deaths globally. Therefore, to make malaria elimination possible, we offer 2 recommendations based on our observations in this study, particularly for tertiary healthcare centers or centers where the burden of severe malaria cases is high. First, malaria elimination efforts will need to include strategies for malaria elimination in humans as well as animal reservoirs. Second, efforts toward the development of novel diagnostics for malaria must be renewed, and AFI diagnoses must include screening for all 5 Plasmodium species, Leptospira, and all 4 DENV serotypes.

This work was funded by the Science and Engineering Research Board Early Career Research (grant no. ECR/ 2016/000833 to P.A.). R.M. was supported by the Department of Biotechnology for a Research Associate fellowship.

\section{About the Author}

Dr. Mandage is a postdoctoral fellow working in the laboratory of Dr. Acharya in the Department of Biochemistry, All India Institute of Medical Sciences, New Delhi, India.

\section{References}

1. Raina S, Raina RK, Agarwala N, Raina SK, Sharma R. Coinfections as an aetiology of acute undifferentiated febrile illness among adult patients in the sub-Himalayan region of north India. J Vector Borne Dis. 2018;55:130-6. https://doi.org/10.4103/0972-9062.242560

2. Susilawati TN, McBride WJH. Acute undifferentiated fever in Asia: a review of the literature. Southeast Asian J Trop Med Public Health. 2014;45:719-26.

3. Joshi R, Kalantri SP. Acute undifferentiated fever: management algorithm [cited 2020 May 29]. http:/ /apiindia. org/wp-content/uploads/pdf/monograph_2015_update_ on_tropical_fever/001_acute_undifferentiated_fever.pdf

4. Ahmad S, Dhar M, Mittal G, Bhat NK, Shirazi N, Kalra V, et al. A comparative hospital-based observational study of mono- and co-infections of malaria, dengue virus and scrub typhus causing acute undifferentiated fever. Eur J Clin Microbiol Infect Dis. 2016;35:705-11. https:/ / doi. org/10.1007/s10096-016-2590-3

5. Salam N, Mustafa S, Hafiz A, Chaudhary AA, Deeba F, Parveen S. Global prevalence and distribution of coinfection of malaria, dengue and chikungunya: a systematic review. BMC Public Health. 2018;18:710. https:/ / doi.org/10.1186/ s12889-018-5626-Z

6. Manock SR, Jacobsen KH, de Bravo NB, Russell KL, Negrete M, Olson JG, et al. Etiology of acute undifferentiated febrile illness in the Amazon basin of Ecuador. Am J Trop Med Hyg. 2009;81:146-51. https:// doi.org/10.4269/ ajtmh.2009.81.146

7. Capeding MR, Chua MN, Hadinegoro SR, Hussain IIHM, Nallusamy R, Pitisuttithum P, et al. Dengue and other common causes of acute febrile illness in Asia: an active surveillance study in children. PLoS Negl Trop Dis. 2013;7:e2331. https:/ / doi.org/10.1371/journal.pntd.0002331 
8. Mueller TC, Siv S, Khim N, Kim S, Fleischmann E, Ariey F, et al. Acute undifferentiated febrile illness in rural Cambodia: a 3-year prospective observational study. PLoS One. 2014;9:e95868. https://doi.org/10.1371/ journal.pone.0095868

9. Chipwaza B, Mugasa JP, Selemani M, Amuri M, Mosha F, Ngatunga SD, et al. Dengue and chikungunya fever among viral diseases in outpatient febrile children in Kilosa district hospital, Tanzania. PLoS Negl Trop Dis. 2014;8:e3335. https://doi.org/10.1371/journal.pntd.0003335

10. Chrispal A, Boorugu H, Gopinath KG, Chandy S, Prakash JAJ, Thomas EM, et al. Acute undifferentiated febrile illness in adult hospitalized patients: the disease spectrum and diagnostic predictors - an experience from a tertiary care hospital in South India. Trop Doct. 2010;40:230-4. https:/ / doi.org/10.1258/td.2010.100132

11. Singh R, Singh SP, Ahmad N. A study of etiological pattern in an epidemic of acute febrile illness during monsoon in a tertiary health care institute of Uttarakhand, India. J Clin Diagn Res. 2014;8:MC01-03. https:// doi.org/10.7860/ JCDR/2014/8965.4435

12. Lindo J, Brown PD, Vickers I, Brown M, Jackson ST, Lewis-Fuller E. Leptospirosis and malaria as causes of febrile illness during a dengue epidemic in Jamaica. Pathog Glob Health. 2013;107:329-34. https:/ / doi.org/10.1179/ 2047773213Y.0000000112

13. Mørch K, Manoharan A, Chandy S, Chacko N, Alvarez-Uria G, Patil S, et al. Acute undifferentiated fever in India: a multicentre study of aetiology and diagnostic accuracy. BMC Infect Dis. 2017;17:665. https:/ / doi.org/ 10.1186/s12879-017-2764-3

14. Barua A, Yeolekar ME. Concurrent dengue and malaria coinfection: observations from a central Mumbai hospital. Int J Infect Dis. 2016;45:165. https:/ / doi.org/10.1016/ j.ijid.2016.02.393

15. Arya SC, Mehta LK, Agarwal N, Agarwal BK, Mathai G, Moondhara A. Episodes of concurrent dengue and malaria [cited 2020 May 29]. https://apps.who.int/iris/bitstream/ handle/10665/164116/dbv29p208.pdf;jsessionid=B3A6898D EDC1ECA2FB605F977C3F0E6E? sequence=1

16. Mushtaq M, Qadri MI, Rashid A, Bin, Qadri MI, Rashid A. Concurrent infection with dengue and malaria: An unusual presentation. Case Rep Med. 2013;2013:1-2. https:/ / doi.org/ 10.1155/2013/520181

17. Barua A, Gill N. A comparative study of concurrent dengue and malaria infection with their monoinfection in a teaching hospital in Mumbai. J Assoc Physicians India. 2016;64:49-52.

18. Carme B, Matheus S, Donutil G, Raulin O, Nacher M, Morvan J. Concurrent dengue and malaria in Cayenne Hospital, French Guiana. Emerg Infect Dis. 2009;15:668-71. https://doi.org/10.3201/eid1504.080891

19. Kaushik RM, Varma A, Kaushik R, Gaur KJBS. Concurrent dengue and malaria due to Plasmodium falciparum and P. vivax. Trans R Soc Trop Med Hyg. 2007;101:1048-50. https://doi.org/10.1016/j.trstmh.2007.04.017

20. Alam A, Dm M. A case of cerebral malaria and dengue concurrent infection. Asian Pac J Trop Biomed. 2013;3:416-7. https:/ / doi.org/10.1016/S2221-1691(13)60087-8

21. Choi JH, Cheong TC, Ha NY, Ko Y, Cho CH, Jeon JH, et al. Orientia tsutsugamushi subverts dendritic cell functions by escaping from autophagy and impairing their migration. PLoS Negl Trop Dis. 2013;7:e1981. https:/ / doi.org/10.1371/ journal.pntd.0001981

22. Frosch AEP, John CC. Immunomodulation in Plasmodium falciparum malaria: experiments in nature and their conflicting implications for potential therapeutic agents.
Expert Rev Anti Infect Ther. 2012;10:1343-56. https:/ /doi.org/ 10.1586/eri.12.118

23. Anvikar AR, Shah N, Dhariwal AC, Sonal GS, Pradhan MM, Ghosh SK, et al. Epidemiology of Plasmodium vivax malaria in India. Am J Trop Med Hyg. 2016;95(Suppl):108-20. https:/ / doi.org/10.4269/ajtmh.16-0163

24. Sharma VP, Dev V, Phookan S. Neglected Plasmodium vivax malaria in northeastern states of India. Indian J Med Res. 2015;141:546-55.

25. van Hellemond JJ, Rutten M, Koelewijn R, Zeeman AM, Verweij JJ, Wismans PJ, et al. Human Plasmodium knowlesi infection detected by rapid diagnostic tests for malaria. Emerg Infect Dis. 2009;15:1478-80. https:/ / doi.org/10.3201/ eid1509.090358

26. Kaur C, Pramanik A, Kumari K, Mandage R, Dinda AK, Sankar J, et al. Renal detection of Plasmodium falciparum, Plasmodium vivax and Plasmodium knowlesi in malaria associated acute kidney injury: a retrospective case-control study. BMC Res Notes. 2020;13:37. https:/ / doi.org/10.1186/ s13104-020-4900-1

27. Pollard TJ, Johnson AEW, Raffa JD, Mark RG. tableone: An open source Python package for producing summary statistics for research papers. JAMIA Open. 2018;1:26-31. https://doi.org/10.1093/jamiaopen/ooy012

28. Rao MRK, Padhy RN, Das MK. Prevalence of dengue viral and malaria parasitic co-infections in an epidemic district, Angul of Odisha, India: an eco-epidemiological and crosssectional study for the prospective aspects of public health. J Infect Public Health. 2016;9:421-8. https:/ / doi.org/ 10.1016/j.jiph.2015.10.019

29. Borkakoty B, Jakharia A, Biswas D, Mahanta J. Co-infection of scrub typhus and leptospirosis in patients with pyrexia of unknown origin in Longding district of Arunachal Pradesh in 2013. Indian J Med Microbiol. 2016;34:88-91. https:/ / doi.org/10.4103/0255-0857.174116

30. Chau JY, Tiffany CM, Nimishakavi S, Lawrence JA, Pakpour N, Mooney JP, et al. Malaria-associated L-arginine deficiency induces mast cell-associated disruption to intestinal barrier defenses against nontyphoidal Salmonella bacteremia. Infect Immun. 2013;81:3515-26. https:/ / doi.org/10.1128/IAI.00380-13

31. Kumar N, Pande V, Bhatt RM, Shah NK, Mishra N, Srivastava B, et al. Genetic deletion of HRP2 and HRP3 in Indian Plasmodium falciparum population and false negative malaria rapid diagnostic test. Acta Trop. 2013;125:119-21. https://doi.org/10.1016/j.actatropica.2012.09.015

32. Pati P, Dhangadamajhi G, Bal M, Ranjit M. High proportions of $p$ fhrp 2 gene deletion and performance of HRP2-based rapid diagnostic test in Plasmodium falciparum field isolates of Odisha. Malar J. 2018;17:394. https:/ / doi.org/10.1186/ s12936-018-2502-3

33. Berhane A, Anderson K, Mihreteab S, Gresty K, Rogier E, Mohamed S, et al. Major threat to malaria control programs by plasmodium falciparum lacking histidine-rich protein 2, Eritrea. Emerg Infect Dis. 2018;24:462-70. https://dx.doi.org/10.3201/eid2403.171723

34. Mussa A, Talib M, Mohamed Z, Hajissa K. Genetic diversity of Plasmodium falciparum histidine-rich protein 2 (PfHRP2) and its effect on the performance of PfHRP2-based rapid diagnostic tests. BMC Res Notes. 2019;12:334. https://doi.org/10.1186/s13104-019-4361-6

35. Megnekou R, Djontu JC, Nana BC, Bigoga JD, Fotso M, Fogang B, et al. Accuracy of One Step malaria rapid diagnostic test (RDT) in detecting Plasmodium falciparum placental malaria infection in women living in Yaoundé, Cameroon. Malar J. 2018; 17:450. https://doi.org/10.1186/s12936-018-2595-8 
36. Watson OJ, Sumner KM, Janko M, Goel V, Winskill P, Slater HC, et al. False-negative malaria rapid diagnostic test results and their impact on community-based malaria surveys in sub-Saharan Africa. BMJ Glob Heal. 2019;4:e001582. https:/ / doi.org/10.1136/bmjgh-2019-001582

37. Anstey NM, Grigg MJ. Zoonotic malaria: the better you look, the more you find. J Infect Dis. 2019;219:679-81. https://doi.org/10.1093/infdis/jiy520

38. Grigg MJ, William T, Barber BE, Parameswaran U, Bird E, Piera K, et al. Combining parasite lactate dehydrogenase-based and histidine-rich protein 2-based rapid tests to improve specificity for diagnosis of malaria due to Plasmodium knowlesi and other Plasmodium species in Sabah, Malaysia. J Clin Microbiol. 2014;52:2053-60. https://doi.org/10.1128/JCM.00181-14

39. Figtree M, Lee R, Bain L, Kennedy T, Mackertich S, Urban M, et al. Plasmodium knowlesi in human, Indonesian Borneo. Emerg Infect Dis. 2010;16:672-4. https:/ / doi.org/ 10.3201/eid1604.091624

40. Ehrhardt J, Trein A, Kremsner P, Frank M. Plasmodium knowlesi and HIV co-infection in a German traveller to Thailand. Malar J. 2013;12:283. https://doi.org/10.1186/ 1475-2875-12-283

41. Kantele A, Marti H, Felger I, Müller D, Jokiranta TS. Monkey malaria in a European traveler returning from Malaysia.
Emerg Infect Dis. 2008;14:1434-6. 10.3201/eid1409.080170 https://doi.org/10.3201/eid1409.080170

42. Tyagi RK, Das MK, Singh SS, Sharma YD. Discordance in drug resistance-associated mutation patterns in marker genes of Plasmodium falciparum and Plasmodium knowlesi during coinfections. J Antimicrob Chemother. 2013;68:10818. https://doi.org/10.1093/jac/dks508

43. Huff CG. The primate malarias. Am J Trop Med Hyg. 1972;21:602-3. https://doi.org/10.4269/ajtmh. 1972.21 .602

44. Singh B, Kim Sung L, Matusop A, Radhakrishnan A, Shamsul SSG, Cox-Singh J, et al. A large focus of naturally acquired Plasmodium knowlesi infections in human beings. Lancet. 2004;363:1017-24. https:/ / doi.org/10.1016/ S0140-6736(04)15836-4

45. Mewara A, Sehgal R. Guest commentary: Plasmodium knowlesi-need to diagnose in India. Trop Parasitol. 2017;7:2-4.

Address for correspondence: Pragyan Acharya, Lab 3002, Department of Biochemistry, All India Institute of Medical Sciences, New Delhi 110029, India; email: pragyan.acharya@aiims.edu

\section{EID Podcast: A Worm's Eye View}

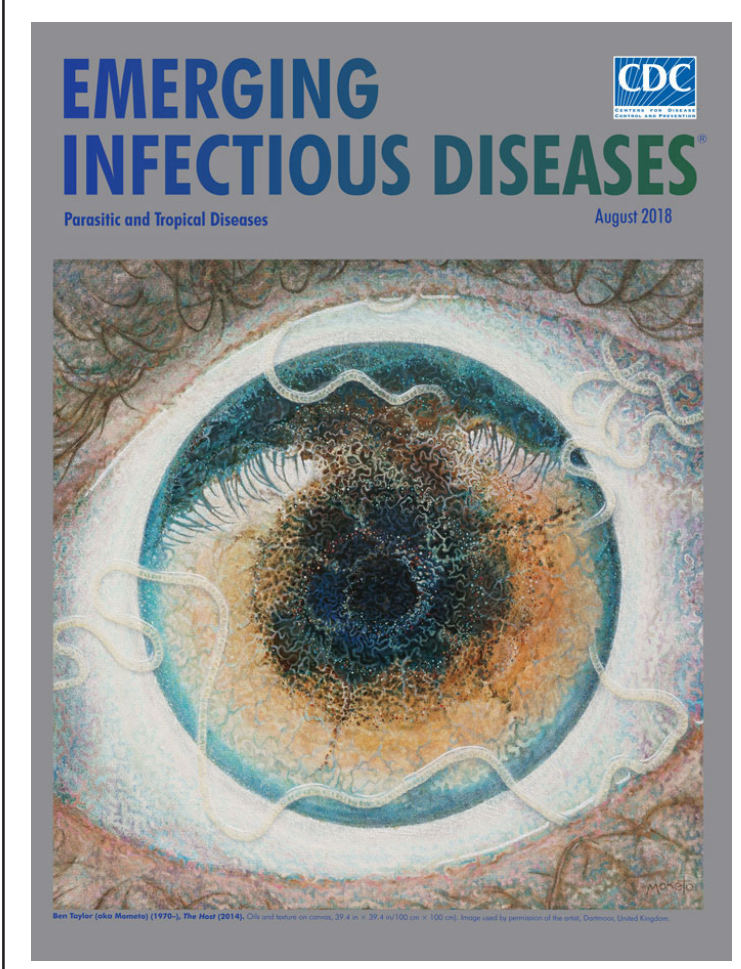

Seeing a several-centimeters-long worm traversing the conjunctiva of an eye is often the moment when many people realize they are infected with Loa loa, commonly called the African eyeworm, a parasitic nematode that migrates throughout the subcutaneous and connective tissues of infected persons. Infection with this worm is called loiasis and is typically diagnosed either by the worm's appearance in the eye or by a history of localized Calabar swellings, named for the coastal Nigerian town where that symptom was initially observed among infected persons. Endemic to a large region of the western and central African rainforests, the Loa loa microfilariae are passed to humans primarily from bites by flies from two species of the genus Chrysops, C. silacea and C. dimidiate. The more than 29 million people who live in affected areas of Central and West Africa are potentially at risk of loiasis.

Ben Taylor, cover artist for the August 2018 issue of EID, discusses how his personal experience with the Loa loa parasite influenced this painting.

\section{Visit our website to listen: https: / /tools.cdc.gov/ medialibrary/index.aspx\# / media/id/392605}

